\title{
Stem Cell Therapy in Liver Cirrhosis
}

Review Article

Ghassemi A ${ }^{1}$, Ommati $\mathrm{H}^{2 *}$, Esmaeilzadeh A , Kooshyar $\mathrm{MM}^{3}$

${ }^{1}$ Associate Professor of Pediatric Hematology \& Oncology \& Stem Cell Transplantation, Hematology and Oncology Ward, Faculty of Medicine, Mashhad University of Medical Sciences.

${ }^{2}$ Gastroenterologist, Shariati Hospital, Mashhad University of Medical Science.

${ }^{3}$ Associate Professor of Hematology \& Oncology, Hematology and Oncology Ward, Faculty of Medicine, Mashhad University of Medical Sciences.

${ }^{4}$ Assistant Professor of Gastroenterology, Internal Medicine Ward, Faculty of Medicine, Mashhad University of Medical Sciences.

\begin{abstract}
Cirrhosis results from different mechanisms of liver injury that lead to necroinflammation and fibrogenesis; Patients with liver cirrhosis often require liver transplantation but it is affected by many problems, including relative operative damage, high costs, lack of donors, and risk of rejection. Currently studies are shown the Stem cell therapy has the potential to provide a valuable adjunct to the management of disease, Stem cell should be the natural candidates to provide a renewable source of cells for transplantation. The main mechanism of stem cell therapy is that stem cell capacity to differentiate into any of the hundreds of distinct cell types that comprise the human body. In addition to their potential in therapeutics can be used to study the earliest stages of human development and disease modeling using human cells. In this article, we review the potential for stem cell therapies to treat liver cirrhosis, MSCs cells are reprogrammed as useful sources of cells that can be change to liver cell for treatment of cirrhosis but pathological implication and autologous bone marrow stem cell therapy for cirrhosis are still to be elucidated and clinical trials that required before this cell transplantation must be performed in Large-scale controlled to becomes a regular therapy.
\end{abstract}

Keywords: Cell Therapy; Liver Cirrhosis; Stem Cell; Transplantation.

\section{Introduction}

The main causes of liver cirrhosis among humans worldwide is chronic viral hepatitis and alcohol abuse that led to the mesenchymal inflammatory and primary disturbance of the hepatic cells, Eventually causes liver cirrhosis [1]. Patients with liver cirrhosis often require liver transplantation but it is affected by many problems, including relative operative damage, high costs, lack of donors, and risk of rejection [2] and because of the increasing shortage of livers for transplantation, Scientists attempted to performed experiments for optimizing and demonstrate the supposed ability of adult bone marrow stem cells to differentiate into mature cells other than blood cells in different adult tissues and functioning hepatocytes [3].
Currently novel studies are shown the Stem cell therapy has the potential to provide a valuable adjunct to the management of cirrhosis, Bone marrow stem cells (BMSCs) have been known to possess the unique capacity for self-renewal and differentiation into hematopoietic and mesenchymal cell lineages [4]. The other reports have demonstrated that BM-derived hepatocytes generated by in vivo cell fusion as well as adult cells can adopt the phenotype of other cell lines by fusing with stem cells [5-7]. However, Stem cell therapy is necessary to consider that the liver already contains endogenous populations of stem cells in hepatocytes that they are able to self-renew almost limitlessly, and often play the principal role in liver regeneration [8].

The aims of this article is to survey the role of stem cell therapies to improve liver cirrhosis and MSCs cells are reprogrammed as

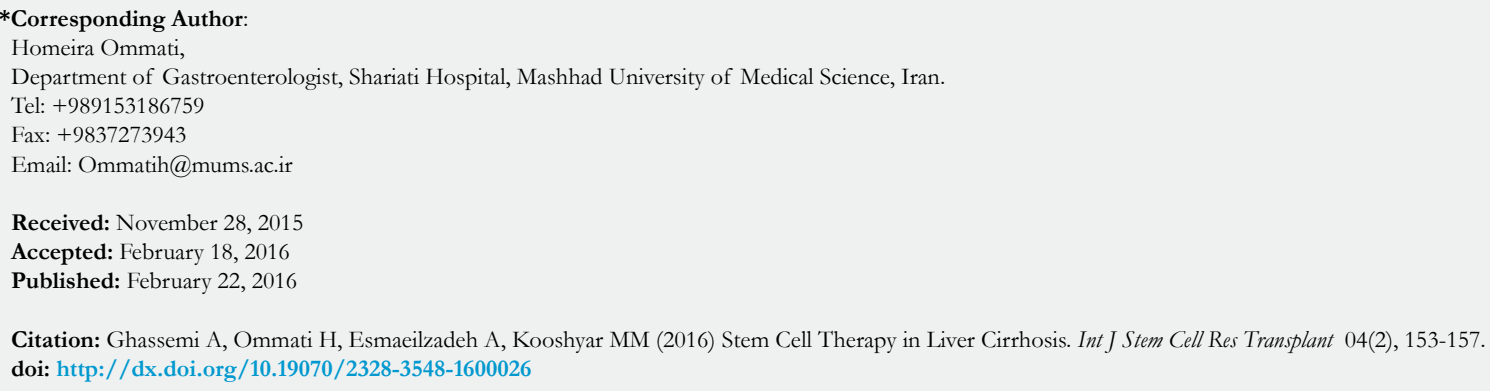

Copyright: $\mathbf{O m m a t i} \mathbf{H}^{\circ}$ 2016. This is an open-access article distributed under the terms of the Creative Commons Attribution License, which permits unrestricted use, distribution and reproduction in any medium, provided the original author and source are credited. 
useful sources of cells with hepatic potential for cirrhosis therapy.

\section{Stem cell}

Stem cells is applied to cells from many different sources with many different functions and phenotypes and is a general descriptive term, Stem cells characteristics are regenerate identical cells, transplant ability, maintain the ability to proliferate and multipotentiality, but can be stimulated to leave differentiate into specific cell types and this self renewal cycle. Stem cells are divided four categories: totipotent, pluripotent, multipotent or unipotent. Totipotent cells are capable of giving rise to any cell in the embryo and extra embryonic tissue. Stem cell populations that may give rise to only limited cell types are multipotent. Cells that give rise to only one cell type are termed unipotent $[9,10]$ and pluripotent is a cell that can generate cell types from each of the three embryonic germ layers: the mesoderm, ectoderm and endoderm [11]. Progenitor (stem) Cell indicates the cells can be dividing with the capacity to differentiate. Every progenitor cell could give rise to a progeny composed of transit-amplifying cells fated for differentiation or initially not committed and retaining self-renewal capabilities [12]. Liver progenitor cells were known as oval cells in rodents. First described for Oval cells were by Farber [13] in 1956 as 'small oval cells with scant lightly basophilic cytoplasm and pale blue-staining nuclei' with a high nuclear/cytoplasmic ratio and an ovoid nucleus. Now oval cells are known as heterogeneous population of bipotential and activate in the liver as a result of injury or insult, but is greatly increased in response to specific types of injury $[13,14]$.

Bone marrow contains Mesenchymal stem cells (MSC) progenitor cells of some mesenchymal tissues, such as cartilage, bone, muscle and fat [14]. MSCs are multipotent cells with the capacity to differentiate to produce multiple types and to promote angiogenesis, clinical studies currently focus on MSC, that are being explored to treat inflammation and regenerate damaged tissue [15]. Bone marrow mesenchymal cells (BMSCs) are thought to be useful for reconstructing injured tissues such as cardiac muscle, bone, or cartilage [16]. Kafienah et al. [17] and Scharstuhl et al. [18] have reported that sufficient numbers of BMSCs with adequate differentiation potential can be used for treatment patients. In animal models, infusion of BMSCs has been shown to engraft in portal tract areas and has been effective in improving survival in rodent models of chronic liver failure [19]. On the other studies, Stem cells have been shown to engraft in portal tract areas, peripheral vein and portal vein infusion has demonstrated high first-pass stem cell entrapment such as hepatic engraftment has focused to use of BMSCs for optimizing [20], However the delivery of BMSCs as cell therapies to the patients could be proven to be an appropriate starting point for treatment of patients [21].

\section{Liver cirrhosis}

Cirrhosis results from variable mechanisms of liver injury that lead to necroinflammation and fibrogenesis, characterized by parenchymal damage [22]. Cirrhosis is $14^{\text {th }}$ most common cause of death in adults worldwide, The main causes in more developed countries are alcohol misuse, infection with hepatitis $C$ virus [23] and the most common cause in sub Saharan Africa and most parts of Asia is infection with hepatitis B virus [24]. Cirrhosis result from a uniform processing developed over several different pathways and a primary mesenchymal inflammatory or primary disturbance of the hepatic cells [25]. Morphological features of cirrhosis include: regenerative nodules, altered lobular architecture, establishment of intrahepatic vascular shunts and diffuse fibrosis, vascular thrombosis and obliterative lesions in portal tracts and hepatic veins, capillarization of sinusoids and perisinusoidal fibrosis and consequent tissue hypoxia [26-28].

Patients with cirrhosis should be treated when possible for the underlying liver disease to stop disease progression; such treatment includes: Drugs for inhibit hepatic fibrosis, immuno suppression for autoimmune hepatitis, antioxidant are treating liver disease caused by chronic alcohol use, phelebotomy for haemochromatosis, copper chelators or zinc for Wilson's disease, when all else fails and the liver is severely damaged, the only treatment is a liver transplantation $[2,29]$ and newly Stem cell therapy has the potential to provide a valuable adjunct to the management of disease, Stem cell should be the natural candidates to provide a renewable source of cells for transplantation [30]. In some literature, bone marrow is suggested as a transplantable source of hepatic progenitors [30] and transplanted BMSC contribute to several non-haematopoietic adult cell types to demonstrate that these BMSC-derived lineages are functional, with a clonogenic capacity, and not merely a transdifferentiated phenotype for cell therapy of cirrhosis [31].

\section{Stem cell in cirrhosis}

Currently, Most of studies have been performed in rodent models of liver injury using either whole/fractionated bone marrow transplantation/injection or peripheral blood stem cells obtained through the administration of growth factors, because of the increasing shortage of livers for transplantation, Scientists attempted to performed experiments for optimizing and demonstration of the supposed ability of adult bone marrow stem cells to differentiate into mature cells other than blood cells in different adult tissues and functioning hepatocytes [3].

In the bone marrow, two major types of adult stem cell are found: mesenchymal stem cells (MSCs) and hematopoietic stem cells (HSCs) [32]. In the previous studies reported that bone marrow isolation and characterization for human clinical trials [28]. MSC progenitor cells are derived from various BM stromal cell types and they produced epithelial cells in a broad range of normal tissues, including the liver [33] and they play a major role in the migration, homing, differentiation, proliferation, and survival of many cell types including human [34]. Aziz et al. [35], Zhao et al [36] and Fang et al. [37] reported in animal models of liver fibrosis, MSC have antifibrosis effects in injured liver has been clearly and MSC have a significant impact on hepatic fibrogenesis through their ability of inhibiting activated HSC and re-regulating the fibrogenic process. Wang et al. demonstrated that the new healthy liver cells in the transplanted $f a b-/-$ mouse contain chromosomes from both recipient and donor cells, with the donor haematopoietic cell nuclei being reprogrammed when they fused with the unhealthy $f a b-/-$ hepatocyte nuclei creating functional hepatocytes [7] and on the surface MSCs expression of CD34 is largely used in clinical practice in human beings and a more stem cell enriched subpopulation of the CD34 cells is expression of CD133 [38]. For isolation of MSCs are more difficult to define the surface expression and is not used routinely [39]. Stem 
cell selection for human clinical trials will reflect of mechanism that including transdifferentiation, stimulation of endogenous hepatocyte proliferation, and antifibrotic and immunomodulatory effects [8].

MSC-based therapies for the treatment of cirrhotic liver disease are essential the gene signaling pathways in hepatic differentiation, stem cell-derived hepatocytes will need to express the genes found in mature liver cells and the level of the expression need to beat or near those found in the normal liver [40]. Recently for demonstrated the hepatic differentiation of human tissue derived MSCs utilized found evidence of transdifferetiation through mesenchymal epithelial transition in the process of hepatic differentiation and microarray analysis to identify the genes responsible for hepatic differentiation [41], also they could be identified hepatic-differentiated cells by the expression of hepatocyte-specific genes [42].

Based on the previous clinical trials, following considerations should be addressed during clinical trials for cirrhotic patients: [1] Utilization of purified MSC population and in addition, their easy accessibility and quick in vitro expansion make MSC an ideal resource for clinical use, thus successfully isolated and in vitro expanded MSC [43]. MSC isolated from patients share the same surface markers and similar biological characteristics to those isolated from healthy humans [40]; [2] MSC passage, During culture period MSC retain cytogenetic stability, and enough number of cells can be obtained for transplantation starting from 10 to $15 \mathrm{ml}$ of bone marrow aspirate [44]; [3] MSC delivery route, Intravenous infusion is the first choice under most circumstances. Although portal vein or hepatic artery delivers may enhance MSC homing efficacy to cirrhotic patients; [4] Evaluation standard, In order to objectively assess the therapeutic effect of MSC transplantation, a standardized criterion must be established prior to MSC administration [40]. Therefore, for identify the complex network of interaction between gene signals during hepatic differentiation are required more studies to facilitate the development of novel methods of therapeutic intervention in human liver cirrhosis.

\section{RTC}

One option for liver cirrhosis is orthotopic liver transplant action and of the increasing shortage of donor liver grafts and the need for long term immunosuppression after liver transplant obviates the need for alternative treatment approaches [45]. Scientists have been identified in liver biopsies after mismatched liver transplantation; they derived bone marrow cells to convert into unexpected cell types in response to tissue stress $[46,47]$. The aims investigated the feasibility, safety, and efficacy of using autologous MSCs as a treatment for liver cirrhosis.

Yannaki et al. reported two cases treated with boost infusions of autologous mobilized hematopoietic stem cells (HSCs) to regenerate cirrhotic liver. Patient 1 (P1) and Patient 2 (P2) were 59 and 57 years old, respectively. They were hospitalized and boost infusions of autologous mobilized hematopoietic stem cells (HSCs) used for them. Both patients showed a lasting amelioration in the clinical course of the disease during the 30 months of follow-up. These results have shown that infusion of mobilized HSCs or mobilization only in a patient with end-stage cirrhosis is a feasible procedure which potentially helps to support liver function as a bridging therapy until liver transplantation becomes available. This could increase the frequency of stem cells engraftment into the liver by potentially facilitating the hominid mechanisms, thus providing a higher beneficial effect to the patients [48].

Peng et al. investigated in 527 patients with liver failure caused by hepatitis B and treated them with a single transplantation with autologous MSCs. Levels of Alb, TBILI, and PT and MELD score of patients in the transplantation group were markedly improved from 2-3 weeks after transplantation, compared with those in the control group. Autologous MSC transplantation is safe for liver failure patients caused by chronic hepatitis B. Short-term efficacy was favorable, but long-term outcomes were not markedly improved. In respect to several parameters, this method is preferable for patients with liver cirrhosis and may have potential for reducing their incidence of hepato cellular carcinom (HCC) and mortality. However in vitro proliferation of autologous MSCs and multiple transplantations with MSCs with high purity and high density may be the key factors for improving the efficacy of transplantation [49].

Kharaziha et al. reported the feasibility, safety, and efficacy of using MSCs as a treatment for eight patients (four hepatitis B, one hepatitis $\mathrm{C}$, one alcoholic, and two cryptogenic) with end-stage liver disease. Approximately, 30-50 million MSCs were proliferated and injected into peripheral or the portal vein. Results Treatment was showed that MSCs injection can be used for the treatment of end-stage liver disease with satisfactory tolerability and to demonstrate the full therapeutic value of this protocol, comprehensive long-term case control clinical needed more studies [50].

El-Ansary et al. demonstrated the effect of autologous transplantation of BM-derived undifferentiated and differentiated MSCs in Twenty-five patients with $\mathrm{C}$ cirrhotic patients following chronic hepatitis $\mathrm{C}$ virus infection. Three months after MSCs injection almost all symptoms of chronic liver failure disappeared except ascites was decreased from moderate to mild ascites. This result showed that BM-MSCs may serve as a versatile, readily available, novel source for therapy of liver diseases and Bone marrow MSCs transplantation can be used as a potential treatment for liver cirrhosis [51]. In the same study by Terai et al. on 9 patients with decompensated liver cirrhosis [52] and Mohamadnejad et al. on 4 patients with decompensated liver cirrhosis [53] were infused with autologous bone marrow cells into the peripheral vein and the data obtained has confirmed the previous studies.

Mohamadnejad et al. showed promising results for bone marrow MSC transplantation in cirrhosis. A total of 27 patients were enrolled. Of these, 15 patients received MSC and 12 patients received placebo. The absolute changes in Child scores, MELD scores, serum albumin, INR, serum transaminases and liver volumes did not differ significantly between the MSC and placebo groups at 12 months of follow-up. In their study, the number of enrolled patients was limited. This can be considered as a limitation of the study. However, because there were neither statistical nor numerical differences observed in the variables between the two groups, they conclude that infusion of autologous bone marrow MSCs through peripheral vein is probably not effective in decompensated cirrhosis. They suggested randomized controlled trials with higher number of patients to evaluate the efficacy of infusion of MSCs earlier in the course of liver disease, repeated infusion of bone marrow cells through the hepatic artery or por- 
tal vein in cirrhotic patients as a bridge to liver transplantation [54].

Khan et al. performed to determine the safety and tolerability of injecting autologous BMSCs (CD34) into four patients with liver insufficiency. In all the patients, cells were infused via the hepatic artery. Three patients completed 6 months follow-up, whereas one patient completed 1 year follow-up. In this study suggested the safety and efficacy of the autologous bone marrow transplantation through the hepatic artery [55] and Sakaida demonstrated reduction in fibrosis in chemically induced liver cirrhosis following BMC transplantation [56]. From a therapeutic point of view, chronic liver cirrhosis is one of the targets for BMC transplantation might be employed. In this condition, there is excessive deposition of extracellular matrix and hepatocytes necrosis. Nikeghbalian et al. reported on six patients with end stage liver disease who were subjected to intraportal administration of autologous bone marrow-derived $\mathrm{CD}_{133^{+}}$in comparison to mononuclear cells in short-term (6 months) and long-term (24 months) follow up. Results were showed no adverse effects in any of the patients during the short and long-term follow up period. Moreover, there were no significant alterations of liver function parameters, liver enzymes, serum albumin, creatinine, serum bilirubin and/or liver volume after transplantation of both types of autologous cells in these patients. They suggested a protocol amendment in which boosts of stem cells can be administered intraportally. This could enhance the frequency of stem cells homing into the liver by potentially facilitating the engrafting mechanisms, thus providing a higher beneficial influence to the patients [57].

Salama et al. demonstrated of autologous stem cell CD34 ${ }^{+}$treatment in 48 patients, 36 with chronic end-stage hepatitis C-induced liver disease and 12 with end-stage autoimmune liver disease. For all patients, granulocyte colony-stimulating factor was administered to mobilize their hematopoietic stem cells. There was clinical and biochemical improvement in a large percentage of patients who received the transplantation. These results suggested that autologous $\mathrm{CD} 34^{+}$stem cell transplantation may be safely administered and appears to offer some therapeutic benefit to patients with both viral and autoimmune-induced end-stage liver disease [58], and Yao et al. in the same study on 100 patients with advanced cirrhosis and who had failed to respond to conservative therapy were recruited for transplantation of autologous peripheral blood $\mathrm{CD}_{3}{ }^{+}$stem cells. Fifty-two patients with massive ascites showed gradual reduction and disappearance of the ascites. Four patients experienced upper gastrointestinal bleeding and three patients developed with hepatic encephalopathy (I-II degree) at 3 months post-transplantation. All patients showed significantly improved liver biosynthesis function, liver elasticity and Knodell score after transplantation. These results showed the autologous peripheral blood $\mathrm{CD} 34^{+}$stem cell transplantation is a safe and effective treatment for advanced cirrhosis, and has high cost-benefit since it improves liver function, liver histology, and quality of life [59].

\section{Conclusion}

Studies suggested that multipotent stem cells may exist among bone marrow cells; and bone marrow stem cells contribute to liver regeneration after injury [54]. In vitro studies reported that bone marrow MSCs can inhibit collagen synthesis and induce apop- tosis in hepatic satellites cells [60] and studies on animal showed the anti-fibrotic effects of MSCs injected through peripheral vein [61]. According to the potential for stem cells to differentiate or their paracrine secretion, stem cell transplantation has become an attractive alternative therapeutic method for the treatment of patients with liver disease, at least, at a temporary support of hepatic function until a liver becomes available for organ transplantation. In this review, the potential of the BMC to differentiate into hepatocytes and other cell lineages has already been reported. Several reports have also demonstrated the plasticity of hematopoietic stem cells to differentiate into hepatocytes, and we demonstrated, MSCs cells are reprogrammed as useful sources of cells with hepatic potential for cirrhosis therapy but pathological implication and autologous bone marrow cell therapy for cirrhosis are still to be elucidated and clinical trials that required before this cell transplantation must be performed in Large-scale controlled to becomes a regular therapy.

\section{Acknowledgments}

We would like to thank all people who participate in this study.

\section{References}

[1]. Lorenzini S, Andreone P (2007) Stem Cell Therapy for Human Liver Cirrhosis: A Cautious Analysis of the Results. Stem Cells 25(9): 2383-2384.

[2]. Tsochatzis EA, Bosch J, Burroughs AK (2014) Liver cirrhosis. Lancet 383(9930): 1749-1761.

[3]. Vig P, Russo FP, Edwards RJ, Tadrous PJ, Wright NA, Thomas HC, et al. (2006) The source of parenchymal regeneration after chronic hepatocellular liver injury in mice. Hepatology 43(2): 316-324.

[4]. Dalakas E, Newsome PN, Harrison DJ, Plevris JN (2005) Hematopoietic stem cell trafficking in liver injury. FASEB J 19(10): 1225-1231.

[5]. Terada N, Hamazaki T, Oka M, Hoki M, Mastalerz DM, et al. (2002) Bone marrow cells adopt the phenotype of other cells by spontaneous cell fusion. Nature 416(6880): 542-545.

[6]. Ying QL, Nichols J, Evans EP, Smith AG (2002) Changing potency by spontaneous fusion. Nature 416(6880): 545-548.

[7]. Wang X, Willenbring H, Akkari Y, Torimaru Y, Foster M, et al. (2003) Cell fusion is the principal source of bone-marrow-derived hepatocytes. Nature 422(6934): 897-901.

[8]. Houlihan DD, Newsome PN (2008) Critical Review of Clinical Trials of Bone Marrow Stem Cells in Liver Disease. Gastroenterology 135(2): 438450.

[9]. Rodriguez-Pinilla SM, Sarrio D, Moreno-Bueno G, Rodriguez-Gil Y, Martinez MA, et al. (2007) Sox2: a possible driver of the basal-like phenotype in sporadic breast cancer. Mod Pathol 20(4): 474-481.

[10]. Jeter CR, Badeaux M, Choy G, Chandra D, Patrawala L, et al. (2009) Functional evidence that the self-renewal gene NANOG regulates human tumor development. Stem Cells 27(5): 993-1005.

[11]. Driesch H (1891) Entwicklungsmechanische Studien I. Der Wert der ersten beiden Furchungszellen in der Echinodermenentwickelung Experimentelle Erzeugung von Teil und Doppelbildungen. Ztschr f Wiss Zool 53: 160-183.

[12]. Farber E (1956) Similarities in the sequence of early histological changes induced in the liver of the rat by ethionine, 2-acetylamino-fluorene, and 3'-methyl-4- dimethylaminoazobenzene. Cancer Res 16(2): 142-148.

[13]. Dabeva MD, Shafritz DA (1993) Activation, proliferation, and differentiation of progenitor cells into hepatocytes in the D-galactosamine model of liver regeneration. Am J Pathol 143(6): 1606-1620.

[14]. Wakitani S, Imoto K, Yamamoto T, Saito M, Murata N, et al. (2002) Human autologous culture expanded bone marrow mesenchymal cell transplantation for repair of cartilage defects in osteoarthritic knees. Osteoarthritis Cartilage 10(3): 199-206.

[15]. Phinney DG, Prockop DJ (2007) Concise review: mesenchymal stem/multipotent stromal cells: the state of transdifferentiation and modes of tissue repair - current views. Stem Cells 25(11): 2896-2902.

[16]. Tomita S, Li RK, Weisel RD, Mickle DA, Kim EJ, et al. (1999) Autologous transplantation of bone marrow cells improves damaged heart function. Circulation 100(19 Suppl II): 247-256.

[17]. Kafienah W, Mistry S, Dickinson SC, Sims TJ, Learmonth I, et al. (2007) 
Three dimensional cartilage tissue engineering using adult stem cells from osteoarthritis patients. Arthritis Rheum 56(1): 177-187.

[18]. Scharstuhl A, Schewe B, Benz K, Gaissmaier C, Buhring HJ, et al. (2007) Chondrogenic potential of human adult mesenchymal stem cells is independent of age or osteoarthritis etiology. Stem Cells 25(12): 3244-3251.

[19]. Gordon MY, Levicar N, Pai M, Bachellier P, Dimarakis I, et al. (2006) Characterisation and clinical application of human CD34+ stem/progenitor cell populations mobilized into the blood by granulocyte colony-stimulating factor. Stem Cells 24(7): 1822-1830.

[20]. Newsome PN, Johannessen I, Boyle S, Dalakas E, McAulay KA, et al. (2003) Human cord blood-derived cells can differentiate into hepatocytes in the mouse liver with no evidence of cellular fusion. Gastroenterology 124(7): 1891-1900.

[21]. Mohamadnejad M, Namiri M, Bagheri M, Hashemi SM, Ghanaati H, et al. (2007) Phase 1 human trial of autologous bone marrow-hematopoietic stem cell transplantation in patients with decompensated cirrhosis. World J Gastroenterol 13(24): 3359-3363.

[22]. Dooley JS, Lok A, Burroughs AK, Heathcote E (2011) Sherlock's diseases of the liver and biliary system. (12

[23]. Lozano R, Naghavi M, Foreman K, Lim S, Shibuya K, et al. (2012) Global and regional mortality from 235 causes of death for 20 age groups in 1990 and 2010: a systematic analysis for the Global Burden of Disease Study 2010. Lancet 380(9859): 2095-2128.

[24]. Blachier M, Leleu H, Peck-Radosavljevic M, Valla DC, Roudot-Thoraval F (2013) The burden of liver disease in Europe: a review of available epidemiological data. J Hepatol 58(3): 593-608.

[25]. Popper H, Elias H (1955) Histogenesis of hepatic cirrhosis studied by the three-dimensional approach. Am J Pathol 31(3): 405-441.

[26]. Millward-Sadler GH, Hanh EG, Wright R (1985) Cirrhosis: an appraisal. Liver and biliary disease. $\left(2^{\text {nd }}\right.$ edtn), Baillière Tindall WB Saunders, London. 821-860.

[27]. Reichen J, Egger B, Ohara N, Zeltner TB, Zysset T, et al. (1988) Determinants of hepatic function in liver cirrhosis in the rat. Multivariate analysis. J Clin Invest 82(6): 2069-2076.

[28]. Wanless IR, Wong F, Blendis LM, Greig P, Heathcote EJ, et al. (1995) Hepatic and portal vein thrombosis in cirrhosis: possible role in development of parenchymal extinction and portal hypertension. Hepatology 21(5): 12381247.

[29]. Morgan TR, Ghany MG, Kim HY, Snow KK, Shiffiman ML, et al. (2010) Outcome of sustained virological responders with histologically advanced chronic hepatitis C. Hepatology 52(3): 833-844.

[30]. Kuo TK, Hung SP, Chuang CH, Chen CT, Shih YV, et al. (2008) Stem Cell Therapy for Liver Disease: Parameters Governing the Success of Using Bone Marrow Mesenchymal Stem Cells. Gastroenterology 134(7): 2111-2121.

[31]. Brittan M, Wright NA (2004) Stem cell in gastrointestinal structure and neoplastic development. Gut 53(6): 899-910.

[32]. Zaitseva M, Kawamura T, Loomis R, Goldstein H, Blauvelt A, et al. (2002) Stromal-derived factor 1 expression in the human thymus. J Immunol 168(6): 2609-2617.

[33]. Lapidot T, Petit I (2002) Current understanding of stem cell mobilization: the roles of chemokines, proteolytic enzymes, adhesion molecules, cytokines, and stromal cells. Exp Hematol 30(9): 973-981.

[34]. Banas A, Teratani T, Yamamoto Y, Tokuhara M, Takeshita F, et al. (2007) Adipose tissue-derived mesenchymal stem cells as a source of human hepatocytes. Hepatology 46(1): 219-228

[35]. Aziz MTA, Atta HM, Mahfouz S, Fouad HH, Roshdy NK, et al. (2007) Therapeutic potential of bone marrow-derived mesenchymal stem cells on experimental liver fibrosis. Clin Biochem 40(12): 893-899.

[36]. Zhao DC, Lei JX, Chen R, Yu WH, Zhang XM, et al. (2005) Bone marrowderived mesenchymal stem cells protect against experimental liver fibrosis in rat. World J Gastroentrol 11(22): 3431-3440.

[37]. Fang B, Shi M, Liao L, Yang S, Liu Y, et al. (2004) Systemic infusion of FLK1+ mesenchymal stem cells ameliorates carbon tetrachloride-induced liver fibrosis in mice. Transplantation 78(1): 83-88.

[38]. Kucia M, Halasa M, Wysoczynski M, Baskiewicz-Masiuk M, Moldenhawer S, et al. (2007) Morphological and molecular characterisation of novel population of CXCR4+ SSEA-4+ Oct-4+ very small embryonic-like cells purified from human cord blood: preliminary report. Leukemia 21(2): 297-303.

[39]. Dominici M, Le Blanc K, Mueller I, Slaper-Cortenbach I, Marini FC, et al. (2006) Minimal criteria for defining multipotent mesenchymal stromal cells. The International Society for Cellular Therapy position statement. Cytotherapy 8(4): 315-317.

[40]. Dai LJ, Li HY, Guan LX, Ritchie G, Zhou JX (2009) The therapeutic po- tential of bone marrow-derived mesenchymal stem cells on hepatic cirrhosis. Stem Cell Res 2(1): 16-25.

[41]. Yamamoto Y, Banas A, Murata S, Ishikawa M, Lim CR, et al. (2008) A comparative analysis of the transcriptome and signal pathways in hepatic differentiation of human adipose mesenchymal stem cells. FEBS J 275(6): 1260-1273.

[42]. Costa RH, Kalinichenko VV, Holterman AX, Wang X (2003) Transcription factors in liver development, differentiation, and regeneration. Hepatology 38(6): 1331-1347.

[43]. Peng L, Li H, Gu L, Peng XM, Huang YS, et al. (2007) Comparison of biological characteristics of marrow mesenchymal stem cells in hepatitis $\mathrm{B}$ patients and normal adults. World J Gastroenterol 13(11): 1743-1746.

[44]. Zhang ZX, Guan LX, Zhang K, Zhang Q, Dai LJ (2008) A combined procedure to deliver autologous mesenchymal stromal cells to patients with traumatic brain injury. Cytotherapy 10(2): 134-139.

[45]. Yannaki E, Athanasiou E, Xagorari A, Constantinou V, Batsis I, et al. (2005) G-CSF-primed hematopoietic stem cells or G-CSF per se accelerate recovery and improve survival after liver injury, predominantly by promoting endogenous repair programs. Exp Hematol 33(1): 108-119.

[46]. Theise N, Nimmakayalu M, Gardner R, Illei PB, Morgan G, et al. (2000) Liver from bone marrow in humans. Hepatology 32(1): 11-16.

[47]. Alison MR, Poulsom R, Jeffery R, Dhillon AP, Quaglia A, et al. (2000) Hepatocytes from non-hepatic adult stem cells. Nature 406(6793): 257.

[48]. Yannaki E, Anagnostopoulos A, Kapetanos D, Xagorari A, Iordanidis F, et al. (2006) Lasting amelioration in the clinical course of decompensated alcoholic cirrhosis with boost infusions of mobilized peripheral blood stem cells. Exp Hematol 34(11): 1583-1587.

[49]. Peng L, Xie D, Lin BL, Liu J, Zhu H, et al. (2011) Autologous Bone Marrow Mesenchymal Stem Cell Transplantation in Liver Failure Patients Caused by Hepatitis B: Short-Term and Long-Term Outcomes. Hepatology 54(3): 820-828.

[50]. Kharaziha P, Hellstrom PM, Noorinayer B, Farzaneh F, Aghajani K, et al. (2009) Improvement of liver function in liver cirrhosis patients after autologous mesenchymal stem cell injection: a phase I-I I clinical trial. Eur J Gastroenterol Hepatol 21(10): 1199-1205.

[51]. El-Ansary M, Aziz IA, Mogawer S, Abdel-Hamid S, Hammam O, et al. (2012) Phase II Trial: Undifferentiated Versus Differentiated Autologous Mesenchymal Stem Cells Transplantation in Egyptian Patients with HCV Induced Liver Cirrhosis. Stem Cell Rev 8(3): 972-981.

[52]. Terai S, Ishikawa T, Omori K, Aoyama K, Marumoto Y, et al. (2006) Improved liver function in patients with liver cirrhosis after autologous bone marrow cell infusion therapy. Stem Cells 24(10): 2292-2298.

[53]. Mohamadnejad M, Alimoghaddam K, Mohyeddin-Bonab M, Bagheri M, Bashtar M, et al. (2007) Phase 1 trial of autologous bone marrow mesenchymal stem cell transplantation in patients with decompensated liver cirrhosis. Arch Iran Med 10(4): 459-466.

[54]. Mohamadnejad M, Alimoghaddam K, Bagheri M, Ashrafi M, Abdollahzadeh L, et al. (2013) Randomized placebo-controlled trial of mesenchymal stem cell transplantation in decompensated cirrhosis. Liver Int 33(1): 1490 1496.

[55]. Khan AA, Parveen N, Mahaboob VS, Rajendraprasad A, Ravindraprakash HR, et al. (2008) Safety and efficacy of autologous bone marrow stem cell transplantation through hepatic artery for the treatment of chronic liver failure: a preliminary study. Transplant Proc 40(4): 1140-1144.

[56]. Sakaida I, Terai S, Yamamoto N, Aoyama K, Ishikawa T, et al. (2004) Transplantation of bone marrow cells reduces $\mathrm{CC} 14$-induced liver fibrosis in mice. Hepatology 40(6): 1304-1311.

[57]. Nikeghbalian S, Pournasr B, Aghdami N, Rasekhi A, Geramizadeh B, et al. (2011) Autologous Transplantation of Bone Marrow derived Mononuclear and CD133+ Cells in Patients with Decompensated Cirrhosis. Arch Iran Med 14(1): 12-17

[58]. Salama H, Zekri AR, Zern M, Bahnassy A, Loutfy S, et al. (2010) Autologous hematopoietic stem cell transplantation in 48 patients with end-stage chronic liver diseases. Cell Transplant 19(11): 1475-1486.

[59]. Yao YT, Luo LY, Xue H, Luo L, Zou HB, et al. (2014) Autologous peripheral blood CD34+ stem cells transplanted into 100 patients with advanced cirrhosis. Zhonghua Gan Zang Bing Za Zhi 22(9): 667-670.

[60]. Parekkadan B, van Poll D, Megeed Z, Kobayashi N, Tilles AW, et al. (2007) Immunomodulation of activated hepatic stellate cells by mesenchymal stem cells. Biochem Biophys Res Commun 363(2): 247-252.

[61]. Zhao W, Li JJ, Cao DY, Li X, Zhang LY, et al. (2012) Intravenous injection of mesenchymal stem cells is effective in treating liver fibrosis. World J Gastroenterol 18(10): 1048-1058. 